FORMATION Formation emploi

Revue française de sciences sociales

97 | janvier-mars 2007

Former pour dynamiser les territoires

\title{
Les politiques de formation dans la structuration des territoires
}

Georgie Simon-Zarca et Michel Vernières

\section{OpenEdition}

1 Journals

Édition électronique

URL : http://journals.openedition.org/formationemploi/1650

DOI : 10.4000/formationemploi.1650

ISSN : 2107-0946

Éditeur

La Documentation française

Édition imprimée

Date de publication : 1 janvier 2007

Pagination : 5-8

ISSN : 0759-6340

\section{Référence électronique}

Georgie Simon-Zarca et Michel Vernières, « Les politiques de formation dans la structuration des territoires », Formation emploi [En ligne], 97 | janvier-mars 2007, mis en ligne le 19 février 2009, consulté le 30 octobre 2020. URL : http://journals.openedition.org/formationemploi/1650 ; DOI : https://doi.org/10.4000/formationemploi.1650

(c) Tous droits réservés 


\title{
Les politiques de formation dans la structuration des territoires
}

\author{
Par Georgie Simon-Zarca et Michel Vernières*
}

La formation est un outil, voire un fer de lance pour le développement des territoires. Voilà un postulat aujourd'hui largement partagé par tous les acteurs de l'emploi et de la formation. Par formation, on entend ici les différentes modalités qui contribuent au développement et au renouvellement des ressources humaines (formation initiale, continue et apprentissage). Mais derrière ce consensus apparent, le rôle et la place que les uns et les autres confèrent à la formation s'inscrivent de fait dans des logiques différentes. Tout d'abord, il apparaît qu'en France, l'appareil de formation est organisé par grands systèmes qui relèvent de cadres institutionnels et d'acteurs différents. Chaque système s'appuie de surcroît sur des relais territoriaux dont le champ d'action se décline différemment. Il en va ainsi de la formation initiale qui relève de l'Éducation nationale et de son découpage académique, de la formation continue des salariés dont l'organisation est étroitement articulée à celle des branches professionnelles, des dispositifs publics de formation destinés aux jeunes sans qualification et aux demandeurs d'emploi dont la prescription relève des niveaux déconcentrés du service public de l'emploi (Agence locale pour l'emploi, Mission locale...).

Cette structuration en tuyau d'orgue des différents systèmes de formation pose des problèmes d'articulation dans les territoires, là où la production de qualification doit être pensée de façon transversale. De ce point de vue, les différentes étapes de la décentralisation favorisent les échanges et la concertation entre les représentants d'institutions porteuses

* Georgie Simon-Zarca est sociologue de formation, chargée de mission Décentralisation et Réseau des Centres associés au Céreq. Ses travaux portent sur les questions de décentralisation et d'évaluation des politiques régionales de formation. Elle a coordonné un ouvrage, Formation et emploi en région: Outils, méthodes, enjeux, travaux du groupe Orafe, Céreq, 2006, 258 p. Elle a co-animé, avec Michel Vernières, un séminaire de travail du réseau des centres associés au Céreq, qui s'était fixé comme objectif de traiter la question des enjeux de la formation pour la dynamique des territoires.

Michel Vernières est professeur émérite d'économie politique à l'université de Paris 1-Panthéon-Sorbonne et chercheur associé au MATISSE-CES - Modélisation appliquée, Transformations institutionnelles et stratégies socioéconomiques/Centre d'économie de la Sorbonne (UMR Paris 7-CNRS). II a longtemps dirigé le Centre associé Céreq pour l'Île-de-France. Ses travaux portent sur les questions d'emploi, analysées en relation avec la formation et les processus de développement (cf. Formation Emploi, enjeu économique et social, Éditions Cujas, 1993). Plus récemment, il a publié : "La formation en tant qu'élément de mobilisation de la main-d'œeuvre », in La mobilisation de la main-d'œeuvre, Michel S., Oudin X. (Éds.), L'Harmattan, juin 2003. "Compétences, qualifications : des constructions d'acteurs multiples », in Solidarités et compétences, idéologies et pratiques, Hours B., Sélim M. (Éds.), l'Harmattan, juin 2003. 
d'objectifs souvent contradictoires. Par exemple, au sein des collectivités territoriales, les acteurs peuvent se positionner tout à fait différemment. Pour certains, la formation constitue un élément central d'un projet de développement économique et social d'un territoire. Pour d'autres, elle est mobilisée dans une perspective d'aménagement, d'équité et de partage entre les territoires.

$\mathrm{Du}$ côté des entreprises, l'enjeu est d'abord de trouver sur le territoire les ressources en maind'œuvre dont elles ont besoin. La nature du tissu économique qu'elles composent collectivement va influer sur les besoins en qualification et le type d'appel au marché du travail. Si cette demande n'est pas satisfaite, les modalités d'interpellation et les formes de collaboration avec les acteurs publics ne seront pas les mêmes d'un territoire à l'autre. Elles seront notamment déterminées par les positions plus ou moins dominantes que certaines entreprises occupent et par le rapport plus ou moins distant qu'elles entretiennent avec chaque système de formation. Les disparités d'accès à la formation continue des salariés selon le secteur d'activité et surtout la taille des entreprises sont, de ce point de vue, particulièrement emblématiques.

Du côté des individus, les entrées de plus en plus tardives sur le marché du travail, la proportion croissante de bacheliers, comme celle des entrées dans l'enseignement supérieur manifestent l'importance que les jeunes et les familles accordent à la poursuite d'études et au diplôme comme réponse aux difficultés rencontrées par les jeunes au cours des premières années de vie active. Cette élévation générale du niveau de formation initiale des jeunes est de bon augure quand on sait l'influence du passé éducatif sur l'accès à la formation continue. Par rapport aux besoins de court terme de l'économie locale, cette élévation peut néanmoins générer des décalages de niveau qui se combinent avec les attentes des jeunes et des familles en matière de choix des filières de formation tant dans le secondaire que dans le supérieur, alors que ces choix ne sont pas forcément conformes à la demande de qualifications adressée par les entreprises.

La relation entre ces trois pôles que constituent la structure de l'offre de formation, la demande de qualifications de la part des entreprises et la demande de formation des individus est au cœur des travaux du Céreq. Depuis la création de cet organisme, la façon de penser cette relation n'a cessé d'évoluer. Elle s'est enrichie peu à peu grâce à l'apport de dimensions nouvelles: effets de conjoncture, rôle des politiques éducatives et d'emploi au niveau national voire européen, politiques de branches, politiques régionales liées à la décentralisation, etc.

L'approche par les territoires, entendus ici comme entités infrarégionales, apporte à la compréhension de cette relation une dimension complémentaire et tout à fait stimulante. Elle contribue à élargir le champ de vision et d'action en incitant à passer d'une relation classique formation-emploi à une relation formation-emploi-développement des territoires. Cette approche mobilise différentes clefs d'entrée. Ainsi, la prise en compte du territoire peut correspondre à un découpage cohérent de l'espace fondé sur la continuité spatiale. L'adoption par le système statistique français d'un découpage du territoire en zones d'emplois et la multiplication des données et analyses produites à ce niveau ont permis, par exemple, de produire des typologies de zones pour mettre en évidence les spécificités des territoires dans une logique comparative. Mais le territoire peut se penser aussi comme un espace d'observation et d'interprétation des processus qui s'y opèrent. Il devient alors un espace qui se définit en fonction du type de réalité qu'on cherche à observer : territoire politique, territoire d'action publique... Quel que soit l'angle d'attaque retenu, construction d'un modèle d'analyse systémique de territoire, analyse d'un système productif local, territoire d'action pour les intermédiaires de l'emploi, territoire comme aire d'attraction des entreprises ou de recrutement d'étudiants, les contributions qui composent ce dossier définissent d'abord le territoire comme un construit social, un espace d'intervention où les systèmes de relations jouent un rôle essentiel.

Formation Emploi a déjà consacré deux dossiers de synthèse aux territoires. Le numéro 84 , dédié aux territoires en formation, mettait tout spécialement l'accent sur les instruments et outils analytiques qui permettent un travail d'experts et facilitent la gouvernance territoriale. Le numéro 87 , construit à partir des données de l'enquête Céreq portant sur l'insertion de la génération 1998, montrait comment les 
territoires façonnent l'insertion professionnelle des jeunes sortant du système éducatif.

Le présent dossier ${ }^{1}$, pour sa part, s'intéresse à la façon dont différents types d'acteurs mobilisent les politiques de formation dans les territoires et contribuent ainsi à les dynamiser. Ces acteurs sont nombreux, mais trois grandes catégories - les entreprises, les institutions de formation et les décideurs politiques - sont principalement prises en compte ici.

Les entreprises, acteurs clés de la structuration productive territoriale, sont au cœur des analyses présentées, y compris les petites et moyennes entreprises, trop souvent négligées dans l'analyse alors qu'elles constituent une part essentielle de la structure productive territoriale.

Les institutions de formation, qu'il s'agisse des universités, des IUT (Institut universitaire de technologie) ou d'autres structures d'enseignement professionnel ont, de par leur présence plus ou moins active et la nature de leurs relations avec les autres acteurs, un impact variable sur la structuration des territoires.

Enfin, les décideurs politiques, qu'il s'agisse de l'État, via ses administrations décentralisées, ou des collectivités locales, se saisissent, à des degrés divers, des mesures mises en place par les politiques nationales de formation et coopèrent plus ou moins harmonieusement et intensément avec les autres acteurs.

D'autres catégories, les familles, les syndicats et les associations, interviennent également localement, pour infléchir les politiques de formation. Leur action ne sera qu'incidemment évoquée.

Limpact de la formation sur les trajectoires territoriales est le résultat de l'interaction de ces divers acteurs, comme l'illustrent les quatre premiers textes de ce dossier qui mettent, à tour de rôle, l'accent sur

${ }^{1}$ Il est le fruit d'une réflexion menée dans le cadre d'un séminaire de travail du réseau des Centres associés au Céreq consacré aux enjeux de la formation pour la dynamique des territoires. Depuis plusieurs années, différents travaux menés le plus souvent dans le cadre d'évaluation de programmes, de dispositifs d'emploi et de formation ou d'expérimentations au niveau local ont approché ces questions. Le groupe de travail pouvait donc s'appuyer sur des matériaux riches qui, de surcroît, mobilisaient des approches disciplinaires diversifiées. Il s'agissait de capitaliser et synthétiser ces travaux afin de dégager des constats transversaux et de progresser collectivement sur des questions de concept, de méthode et de cadre d'analyse. Le dossier présenté ici cherche à rendre compte, pour partie, de cette double ambition. certains d'entre eux, avant que la dernière contribution ne tente une synthèse analytique multidisciplinaire des approches proposées.

L'étude de J. Haas s'appuie sur l'émergence d'un système productif local consacré à la mécanique. Ce type de configuration productive est particulièrement emblématique, depuis Alfred Marshall en passant par les analystes des districts italiens, des analyses des structures productives des territoires. Dans le cas présent, l'intérêt majeur de ce travail est de porter sur un exemple où la politique de formation a joué un rôle clé. Il en ressort l'impact décisif de l'action volontariste et collective des décideurs politiques, des entreprises, en premier lieu de l'entreprise leader de la zone ainsi constituée, et de l'appareil de formation. Cette action a été facilitée par la prise de conscience d'un intérêt commun pour résister à la domination de la métropole régionale et acquérir la main-d'œuvre qualifiée nécessaire. C'est donc la coalition de ces acteurs privés et publics qui a permis la création d'un IUT spécialisé dans les domaines souhaités et le développement de la formation en alternance. Cet IUT, une fois créé, a joué un rôle significatif dans la diffusion locale d'une technologie innovante, ce qui souligne l'importance sur un territoire des structures de formation et de recherche.

Ce rôle des institutions universitaires de recherche pour la caractérisation de la structure des territoires est particulièrement mis en lumière par le travail de R. Levy et R. Woessner portant sur la place, dans les régions, de la formation par la recherche conduite en partenariat avec les entreprises. Les auteurs utilisent les données relatives aux bourses «CIFRE » (conventions industrielles de formation par la recherche) qui permettent à de jeunes chercheurs de préparer leur doctorat sur un sujet et dans des conditions déterminés conjointement par un laboratoire universitaire et une entreprise. Comme cela était prévisible, il apparaît une forte centralisation des relations, polarisées sur la région parisienne. Néanmoins, en moyenne, environ $30 \%$ de ces contrats sont passés entre entreprises et laboratoires d'une même région, ce qui n'est pas sans influence sur la structuration productive de cette dernière. Mais on observe de nettes différences dans les dynamiques territoriales d'un espace à l'autre. Ces résultats confortent la pertinence d'une approche territoriale, 
même dans le cas limite de formations de haut niveau qui ont une dimension nationale et internationale marquée. De plus, la mise en évidence de «grappes urbaines », constituant des réseaux régionaux ou en lien avec Paris, invite à souligner que l'approche en termes de réseaux est essentielle pour l'analyse territorialisée des questions de formation.

Cette importance analytique et opérationnelle des réseaux avait déjà, brièvement, été soulignée dans le cas, en apparence bien différent, des politiques de formation des PME (petites et moyennes entreprises), analysées, dans le dossier du numéro 84, par E. Bentabet et S. Michun. Ici, ce dernier développe et prolonge leur analyse. Il montre que l'ancrage local de ces entreprises n'est qu'un élément susceptible d'expliquer leur comportement en matière de formation. Plus importante apparaît la prise en compte des types de réseaux dans lesquels elles s'inscrivent. Ainsi les entreprises qu'il qualifie de « traditionnelles » s'insèrent essentiellement dans des réseaux d'affaires, les entreprises «managériales» dans des réseaux hiérarchiques. Quant aux entreprises «néo-libérales», elles participent à des réseaux de projet. Dès lors, selon que les territoires considérés comprennent plus ou moins de PME de ces divers types, les modalités de gestion de la formation de leur main-d'œuvre vont varier. Les autorités chargées des politiques de formation vont devoir tenir compte de cette diversité pour rendre plus efficaces ces politiques et choisir les instruments adaptés aux comportements différenciés de ces firmes.

Létude présentée par M. Lecoutre, complémentaire de la précédente, examine les modalités d'une politique publique d'incitation à la formation dans les PME, les «engagements de développement de la formation » (EDDF). Ce travail conduit à la même conclusion: l'auteur montre que cette politique publique, initiée au niveau national, a impérativement besoin de médiateurs territoriaux, d'intermédiaires de proximité. Ceux-ci permettent la mise en oeuvre de mesures qui, pour être effectives et efficaces, doivent être appropriées par les PME locales en fonction de la nature de leur insertion dans les réseaux évoqués plus haut. Dans le cas des EDDF, le ministère du Travail a choisi les branches professionnelles comme intermédiaires. Mais le recours à ces intermédiaires se heurte à deux types de difficultés. D'une part, comme tout acteur, les branches ont leur stratégie propre et peuvent donc détourner la mesure à leur profit, comme cela semble avoir été le cas pour la métallurgie. D'autre part, l'intermédiaire peut ne pas être assez structuré localement pour jouer pleinement son rôle, ce qui est le cas de la grande distribution alimentaire dans les deux régions étudiées. Là encore, la spécificité territoriale apparaît. Selon le type de secteur d'activité dominant et la force relative des intermédiaires mobilisés, le même instrument de politique de formation aura un impact très différent d'un territoire à l'autre.

À la suite de ces quatre approches, réalisées sous des angles particuliers mais complémentaires de la relation entre politiques de formation et structuration des territoires, il a semblé utile de tenter un éclairage conceptuel de ces contributions. M. Bel s'est consacrée à cette tâche difficile. Elle explore, d'un double point de vue, thématique et disciplinaire, plusieurs notions largement présentes dans les différentes contributions. Acteurs, intermédiaires, réseaux, proximité, sont en effet apparus comme des cadres d'analyse puissants pour qualifier et interpréter les interactions complexes entre agents hétérogènes sur un territoire.

Pas plus que les précédents, ce dossier ne prétend faire le tour de la relation formation-emploi-développement dans les territoires. Portée par la dynamique de la décentralisation, cette thématique est encore trop récente pour faire l'objet d'une quelconque synthèse exhaustive. Pourtant, par la complémentarité des éclairages que chaque contribution apporte, ces dossiers esquissent, par touches successives, un champ de recherche prometteur qui ne demande qu'à être exploré plus avant. 